western blot for eNOS signalling proteins and NOS activity assay were undertaken in cirrhotic and control kidneys. Markers of oxidative stress and inflammatory cytokines were measured in serum by ELISA. We studied the effects of s.c. infusion of recombinant human RLN(seralaxin; $72 \mathrm{~h}, 4 \mu \mathrm{g} / \mathrm{h}$ ) on these parameters. Doppler USS measured changes in cardiac output (CO) and renal arterial resistive index (RRI) in response to i.v. RLN $(4 \mu \mathrm{g})$. Kidney endothelial morphology was assessed by electron microscopy, $\mathrm{H}+\mathrm{E}$ and PAS stained kidney by light microscopy.

Results In renal arteries from control and cirrhotic rats endothelial vasodilatation was eNOS-dependent. In cirrhotic rats endothelium-dependent relaxation (acetylcholine; 10-9-3 $\times 10$ $5 \mathrm{M})$ was dramatically reduced $(\mathrm{p}<0.0001)$ in all renal arteries, with only a modest reduction seen in the mesenteric arteries. Endothelium-independent relaxation (sodium nitroprusside; 10-9-3 × 10-5 M) and vasoconstriction (phenylephrine; 10-9-3 × 10-5 M) were unaltered. In cirrhotic kidneys, total eNOS expression was up-regulated,as were arginase 2 and caveolin1 (negative regulators of eNOS), and NOS activity was reduced ( $\mathrm{p}<0.05$ ). Acute RLN had no effect on CO but decreased RRI $(p<0.05)$. Extended RLN restored endotheliumdependent relaxation, increased kidney NOS activity $(\mathrm{p}<0.05)$, increased phosphorylated Akt and eNOS, and reduced serum TNF $\alpha$ levels.

Conclusion Renal vascular endothelial dysfunction characterises experimental cirrhosis, through a reduction in renal eNOS activity. This impairment may contribute to the renal vasoconstriction seen in cirrhosis and is a promising target for therapeutic modulation. RLN treatment restored renal endothelial vasodilatation. The potential for recombinant forms of RLN as a haemodynamic modulator in human cirrhosis and HRS merits investigation in translational studies.

Disclosure of Interest None Declared.

\section{OC-032 SURVEILLANCE LEADS TO IMPROVED OUTCOMES FOR PATIENTS WITH HEPATOCELLULAR CARCINOMA (HCC)}

${ }^{1}$ TJS Cross*, ${ }^{1}$ W Ding, ${ }^{1} P D$ Richardson, ${ }^{1} F$ Yousef, ${ }^{2} D$ Palmer, ${ }^{3} E$ Joekes, ${ }^{3} \mathrm{C}$ Farrell, ${ }^{3}$ J Evans. ${ }^{1}$ Department of Gastroenterology and Hepatology, The Royal Liverpool Hospital, Liverpool, UK; ${ }^{2}$ Academic Department of Oncology, The University of Liverpool, Liverpool, UK; ${ }^{3}$ Department of Radiology, The Royal Liverpool Hospital, Liverpool, UK

\subsection{6/gutjn-2014-307263.32}

Introduction 6-monthly ultrasound surveillance is recommended in cirrhotic patients at risk of HCC. The benefit of surveillance has never been demonstrated in a western population.

Methods A retrospective, single centre cohort analysis in patients diagnosed with HCC from 2008-2013. From 2008 an automated recall system for 6-monthly ultrasound was instigated by the radiology department, in preference ad hoc ultrasound requests. Patients with abnormal lesions proceeded to CT, MRI or liver biopsy according to defined international criteria. The primary end-points evaluated were stage of cancer detection (early i.e. BCLC 0 or A), versus late presentation (BCLC B-D) and patient survival from time of diagnosis to 12 months and 60 months.

Results 160 patients were identified. Surveillance status was known in 132 patients. Median patient age was 68 years (57-75), median number of lesions was one, diameter of largest lesion $30 \mathrm{~mm}$ (19-50), and AFP 19.5 (5-250). Patients under surveillance were more likely to have disease at a curative stage 67 vs. $39 \%(\mathrm{p}=0.006$, OR $0.59(0.41-$ 0.84 ), and had better survival at 1 year 80 vs. $62 \%$ ( $\mathrm{p}=$
0.04 , OR $0.77(0.62-0.97)$, and at 5 years 60 vs. $41 \%(\mathrm{p}=$ 0.046 , OR 0.69 (0.48-0.98). On univariate analysis the following variables on survival were evaluated: Age $(\mathrm{p}=0.11)$, Number HCC nodules $(\mathrm{p}=0.31)$, Total diameter of lesions $(\mathrm{p}=0.001)$, Diameter of largest lesion $(\mathrm{p}<0.001)$, AFP $(\mathrm{p}$ $<0.001)$. The presence on imaging of extra-hepatic metastases $(p=0.006)$, lymph nodes $(p=0.004)$, and portal vein thrombosis $\mathrm{p}<0.001)$, were associated with poorer survival.

Conclusion Surveillance for hepatocellular carcinoma leads to earlier diagnosis and improved survival.

Disclosure of Interest None Declared.

\section{OC-033 THE TWEAK AND FN14 PATHWAY AS POTENTIAL MEDIATOR OF LIVER FIBROSIS}

${ }^{1} \mathrm{~A}$ Wilhelm*, ${ }^{1} \mathrm{M}$ Munir, ${ }^{1} \mathrm{E}$ Humphreys, ${ }^{1} \mathrm{D}$ Adams, ${ }^{2} \mathrm{~L}$ Burkly, ${ }^{1} \mathrm{~S}$ Afford, ${ }^{1} \mathrm{C}$ Weston. ${ }^{1}$ Centre for Liver Research, University of Birmingham, Birmingham, UK; ${ }^{2}$ Biogen Idec, Cambridge, USA

\subsection{6/gutjnl-2014-307263.33}

Introduction The TNF superfamily ligand TWEAK and its cognate receptor Fn14 have been implicated in the pathogenesis of liver disease and have been predominantly associated with liver progenitor cell proliferation and ductular reaction. We hypothesised that TWEAK and Fn14 may also be involved in the establishment and progression of fibrosis via a direct effect on hepatic stellate cell (HSC) function.

Methods TWEAK and Fn14 expression was studied by qPCR, western blot and immunostaining in tissue and stromal cells from explanted human liver specimens and normal donor livers surplus to surgical requirements, or as a byproduct of surgical resection. The responses of HSCs to TWEAK were investigated by western blot, live cell imaging and proliferation assays. TWEAK was measured in HSC supernatant by ELISA.

Results Confocal microscopy revealed localisation of Fn14 to cells expressing stromal markers in normal human livers, with significant upregulation in diseased livers. Fn14 expression was confirmed in both primary human HSCs and myofibroblasts in vitro. Stimulation with recombinant TWEAK led to an upregulation of NF-kB signalling and induced proliferation in cultured HSCs. TWEAK immunostaining localised the protein to the fibrotic areas of ALD and NASH liver sections suggestive of an autocrine regulation of Fn14 signalling. We confirmed that HSCs express TWEAK and release it into their environment by qPCR and ELISA, and demonstrated that function-blocking TWEAK antibodies reduced the proliferative capacity of HSC.

Conclusion Our study suggests that TWEAK/Fn14 promotes liver fibrosis via enhanced proliferation of HSC, possibly through an autocrine mechanism driven by HSC production of TWEAK.

Disclosure of Interest None Declared.

\section{OC-034 OUTCOME OF PATIENTS CONSIDERED UNSUITABLE FOR LIVER TRANSPLANTATION - A MISSED OPPORTUNITY FOR PALLIATIVE CARE?}

A Phoolchund*, S Murray, B Hogan, J O'Beirne. Royal Free Hospital and UCL Institute of Liver and Digestive Health, Royal Free Hospital, London, UK

10.1136/gutjnl-2014-307263.34 